\title{
Patient- and provider-level risk factors associated with default from tuberculosis treatment, South Africa, 2002: a case-control study
}

\author{
Alyssa Finlay ${ }^{1 *}$, Joey Lancaster ${ }^{2}$, Timothy $\mathrm{H} \mathrm{Holtz}^{1}$, Karin Weyer ${ }^{3}$, Abe Miranda ${ }^{1}$ and Martie van der Walt ${ }^{2}$
}

\begin{abstract}
Background: Persons who default from tuberculosis treatment are at risk for clinical deterioration and complications including worsening drug resistance and death. Our objective was to identify risk factors associated with tuberculosis (TB) treatment default in South Africa.

Methods: We conducted a national retrospective case control study to identify factors associated with treatment default using program data from 2002 and a standardized patient questionnaire. We defined default as interrupting TB treatment for two or more consecutive months during treatment. Cases were a sample of registered TB patients receiving treatment under DOTS that defaulted from treatment. Controls were those who began therapy and were cured, completed or failed treatment. Two respective multivariable models were constructed, stratified by history of TB treatment (new and re-treatment patients), to identify independent risk factors associated with default.
\end{abstract}

Results: The sample included 3165 TB patients from 8 provinces; 1164 were traceable and interviewed (232 cases and 932 controls). Significant risk factors associated with default among both groups included poor health care worker attitude (new: AOR 2.1, 95\% Cl 1.1-4.4; re-treatment: AOR 12,95\% Cl 2.2-66.0) and changing residence during TB treatment (new: AOR 2.0, 95\% Cl 1.1-3.7; re-treatment: AOR 3.4, 95\% Cl 1.1-9.9). Among new patients, cases were more likely than controls to report having no formal education (AOR 2.3, 95\% Cl 1.2-4.2), feeling ashamed to have TB (AOR 2.0,95\% Cl 1.3-3.0), not receiving adequate counseling about their treatment (AOR 1.9, 95\% Cl 1.2-2.8), drinking any alcohol during TB treatment (AOR 1.9,95\% Cl 1.2-3.0), and seeing a traditional healer during TB treatment ( $A O R$ 1.9, 95\% Cl 1.1-3.4). Among re-treatment patients, risk factors included stopping TB treatment because they felt better (AOR 21,95\% Cl 5.2-84), having a previous history of TB treatment default (AOR 6.4, 95\% Cl 2.9-14), and feeling that food provisions might have helped them finish treatment (AOR 5.0, 95\% Cl 1.3-19).

Conclusions: Risk factors for default differ between new and re-treatment TB patients in South Africa. Addressing default in both populations with targeted interventions is critical to overall program success.

Keywords: Tuberculosis, treatment default, non-adherence, South Africa

\section{Footnote page}

All authors had access to this data and share responsibility for the decision to submit this manuscript for publication. All authors have provided a description of their contribution and have signed a statement granting exclusive license for publication to BMC Infectious Disease.

\footnotetext{
* Correspondence: avf0@cdc.gov

'Division of Tuberculosis Elimination, Centers for Disease Control and

Prevention, 1600 Clifton Road NE, Atlanta, GA 30333, USA

Full list of author information is available at the end of the article
}

The views expressed within this paper are solely those of the authors and do not reflect those of the U.S. Public Health Service or the South African Medical Research Council. There are no financial, personal or professional interests that could be construed to influence the paper.

Trade names are used for identification only and do not represent endorsement by the Centers for Disease Control and Prevention or the U.S. Public Health Service. 
One or more authors were employees of the US federal government when this work was conducted and prepared for publication; therefore, it is not protected by the Copyright Act, and copyright ownership cannot be transferred.

\section{Background}

In 2002, South Africa ranked 9th worldwide in terms of total number of tuberculosis (TB) cases and 8th in terms of the highest TB incidence rates per capita. The incidence of all reported TB cases had increased 2.5 fold between 1992 (228/100,000) and 2002 (558/100,000) [1]. By 2001, TB was the leading reported cause of death by natural causes [2]. While case detection and implementation of DOTS increased substantially during this time frame, by 2002, treatment success rates remained low (65\% for new cases and 53\% for re-treatment cases), and default (12\% for new cases and $17 \%$ for re-treatment cases) and death (7\% for new cases and 9\% for re-treatment cases) rates high [1].

Persons under treatment for TB who default from treatment are at risk for clinical deterioration and complications, can continue to be infectious to others, and are at risk of premature death from TB [3]. There are limited studies on the risk factors associated with TB treatment default in S. Africa under DOTS [4-7]. Having had a history of TB treatment has been associated with TB treatment default [8-10]. South Africa faces unique challenges with an unparalleled co-epidemic of TB and human immunodeficiency virus (HIV), in the setting of an overburdened health-service sector. There are complex economic and environmental conditions, including high levels of urban migration and unemployment, and unique socio-cultural risk factors that may have important influences on TB treatment adherence. The relative contribution of these factors to the problem of default from TB treatment is not known. We performed a retrospective case-control study to evaluate risk factors for default from TB treatment in 8 of 9 provinces in South Africa in 2002. Our primary objective was to identify patient-level and provider-level risk factors associated with default from TB treatment.

\section{Methods}

We conducted a questionnaire-based, case-control study among adult persons $\geq 18$ years old enrolled in treatment under DOTS at public health facilities in South Africa between January 1 and December 31, 2002. Eligible cases were defined as any new and re-treatment TB patient (with pulmonary or extrapulmonary $\mathrm{TB}$ ) who were on treatment for at least 4 weeks, then defaulted from a six-month (new) or eight-month (re-treatment) TB regimen. New patients received a fixed dose combination Rifafour ${ }^{\circledR}$ (isoniazid, rifampin, pyrazinamide, ethambutol), retreatment cases received Rifafour ${ }^{\circledR}$ plus streptomycin injection.

We defined default as interrupting TB treatment for two or more consecutive months during treatment. Eligible controls were those persons diagnosed with TB who began therapy and were cured, completed or failed treatment. Persons were excluded if they were recorded to have died or transferred, were younger than 18 years, were known to have multidrug-resistant $\mathrm{TB}$ or were prisoners or wards of the state.

Interviewers were trained to review medical records available at the health facilities, and to trace and interview TB patients with written informed consent. Interviewers made every effort to locate patients using information available from the health center records or staff who knew the patients; they telephoned patients, located their homes if possible and made multiple visits if necessary. In the event a patient no longer lived at a specific address, they asked local neighbors and guides to help them trace the patients. To minimize bias, the interviewers did not know the patients and were not associated with the local health services. Data collected from the health facilities included demographic information, patient's address, treatment information including dates of TB registration, treatment initiation and completion and treatment outcome.

A structured questionnaire was adapted from a questionnaire previously used in South Africa to study MDR TB adherence, with additional questions concerning the role of food and nutrition during TB treatment [6]. Questions covered five domains commonly cited regarding adherence to long-term therapies (Figure 1) [11]. The questionnaire was pre-tested and translated from English into 10 South African home languages (Xitsonga, Tshivenda, Siswati, Setswana, Sesotho, IsiZulu, IsiXhosa, IsiNdebele, Afrikaans) and back-translated into English to ensure the quality of translation. The

\section{Domains of Adherence [11]}

- Social and economic factors

- Patient-related factors

- Condition-related factors

- Therapy-related factors

- Health care team and systemrelated factors

Figure 1 Domains of adherence. 
questionnaire was a combination of multiple-choice, yes/no and open-ended questions on demographic, social, health service and treatment characteristics. We inquired about the provision of nutritional support and the role of enablers and incentives. Information on the HIV status of persons with TB was not included as HIV services were not integrated in the TB program in 2002.

Questionnaires were administered in the TB patient's home language using face-to-face interviews. Interviewers were trained to encourage defaulters to return to the health clinic to be evaluated for TB treatment. For patients who were found to have died, the outcome of death, date of death and cause, if available, was recorded. Patients were given a standard food parcel equivalent to approximately US\$15 for participating in the study, offered after the informed consent and interview so as to not bias study participation or responses.

The sample was selected from facility-based national TB registers in 8 provinces and matched by quarter of enrolment and health facility at a ratio of 1 case: 2 controls. TB registers from Limpopo province were not available and thus excluded. Sample selection for each province was conducted by multi-stage sampling made up of urban and rural sub-samples that reflected the estimated demographic population proportions reported for 2001. For each province, we compiled a list of all urban and rural public clinics including number of new patients enrolled and number of defaulters in 2002. The health facility sub-samples were chosen by systematic sampling proportional to clinic enrolment size. TB registers were requested from selected health facilities and the pre-calculated number of cases and matched controls were selected randomly from the TB registers.

Based on historic program data on the proportion of patients from each province classified as re-treatment patients (8-27\% depending on the province), we calculated a sample size large enough to show at least a $25 \%$ difference between cases and controls that would result in a statistically significant odds ratio (OR) of $>2.9$ with a $95 \%$ confidence interval $(\mathrm{CI})$ and a power of $80 \%$, for each province. In anticipation of difficulties associated with finding defaulters, the overall sample size was increased by $75 \%$. Based on these calculations, the targeted sample size for the 8 provinces was 1055 cases and 2110 controls.

Data were entered into an EpiData v.3.1 database and analyzed with STATA $8[12,13]$. Data were validated by checking consistency values, evaluating range values, and performing uniqueness checks. We analyzed differences in proportions using the Mantel-Haenszel chi-square statistic $\left(\chi^{2}\right)$ or Fisher's exact test where appropriate. For data not normally distributed, we compared differences in medians using the Wilcoxon rank-sum test. We performed multivariable logistic regression analysis with forward selection. We evaluated all variables in the bivariate analysis that were significant at $P<0.20$ in the multivariable analysis using logistic regression for the final models. For all statistical tests, we considered a $P$ value of $<0.05$ as statistically significant. All pair wise interactions of the explanatory variables that were epidemiologically relevant in the model were considered.

\section{Ethics statement}

This study was approved by the Ethics Committee of the South African Medical Research Council and the respective provincial research committees. This study was also approved as human subjects' research by the Institutional Review Board at the US Centers for Disease Control and Prevention in Atlanta, GA, USA. Voluntary written informed consent was obtained from all participants included in the study.

\section{Results}

We collected data between July 2004 and August 2005. Of 3165 patients sampled, health records were available for 3079 (97\%). Of these, we successfully traced and interviewed 1164 (232 cases and 932 controls, 26\% and $43 \%$ respectively) (Figure 2 ). Few patients refused to be interviewed (34 patients, 1\%). An additional 1319/3079 (43\%) patients could not be traced, due to incorrect addresses listed in health facility records or were not eligible. A large proportion (18\%) of patients were found to have died, including 210/900 (23\%) cases and 352/ 2179 (16\%) controls $(P<0.001)$. The date of death was known for 115/200 (55\%) cases. Of these, 33 (22\%) were reported to have died within 2 months after stopping TB treatment before completion (no data on when during treatment). These patients were probably misclassified as defaulters, and were likely deaths.

Limited clinical data were available from patients who were not interviewed. Cases interviewed vs. not interviewed were more likely to be younger, to have attended a rural clinic, to be a re-treatment patient, and to default later in the treatment course. Controls interviewed vs. not interviewed were more likely to have attended a rural clinic, to be a re-treatment patient and less likely to have been admitted to an inpatient healthcare facility for TB treatment. Patients who were not interviewed were excluded from further analysis.

We explored factors associated with default, stratified among new and re-treatment patients. Among the 1164 patients interviewed, 2/1164 had an unknown patient category and were excluded from the analysis. Tables 1 , 2 and 3 summarize the main characteristics from the health facility record review and interviews with 1162 patients (231 cases and 931 controls) who had a known patient category, were successfully traced, and consented to participate. 


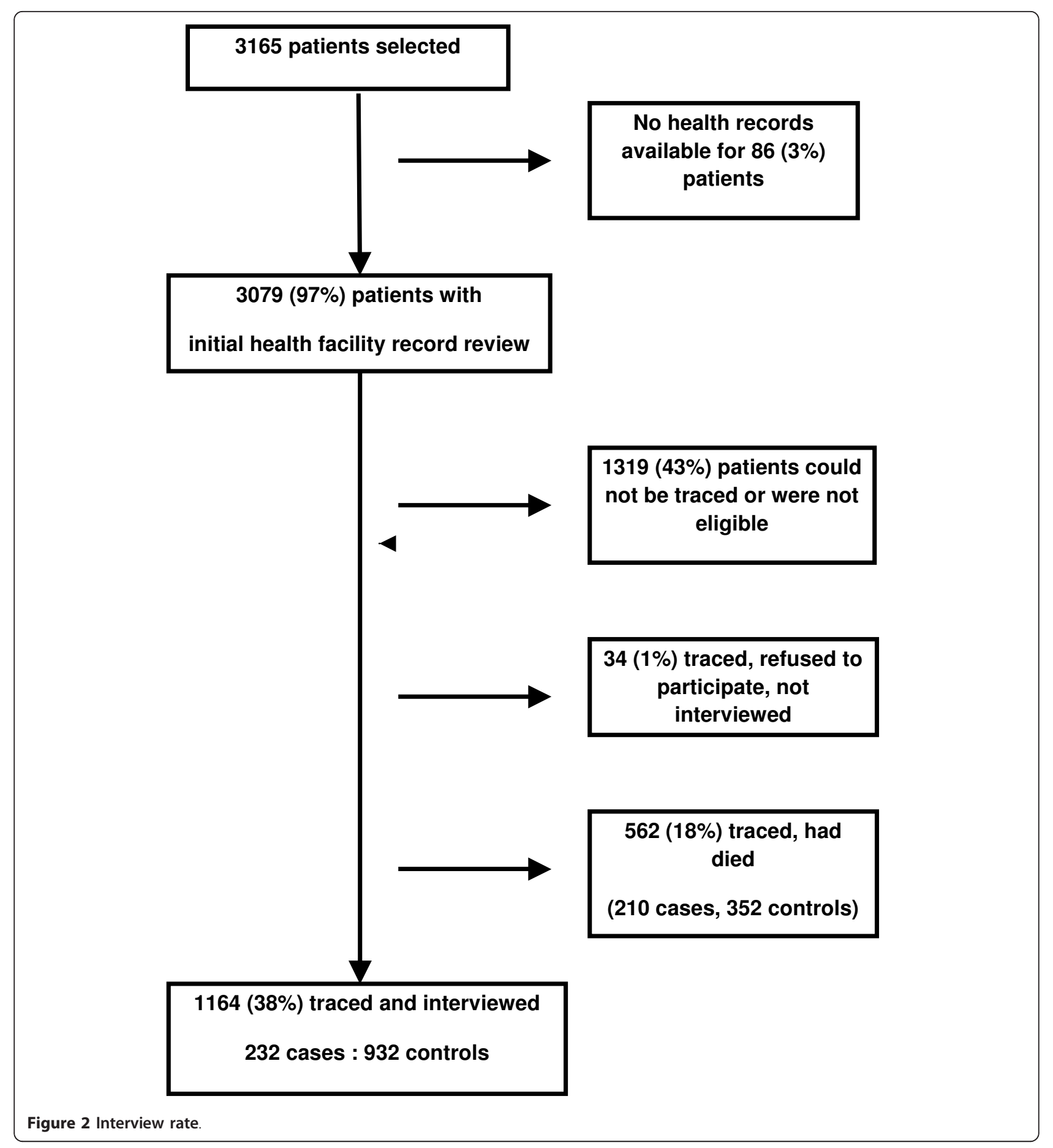

Social and economic characteristics

Table 1 shows the age distribution and sex of patients, stratified by patient category, new and re-treatment patients. Among new patients, cases were more likely to be male (OR 1.5, 95\% CI 1.1-2.2). Among both new and retreatment patients, the median age of cases was younger than controls. Most patients were born in South Africa, single, black African, reported practicing religion, and had one or more children (Table 2). Unemployment was common and similar between groups ( $47 \%$ of cases and 52\% of controls). Among employed patients, default was associated with patients missing treatment due to employment. Reasons cited by patients included that they were too busy and did not have enough time, work was too far from the TB clinic, employer did not allow them to get TB treatment and 
Table 1 Sex and age distribution of 231 cases and 931 controls with TB, stratified by patient category, South Africa, 2002*

\begin{tabular}{|c|c|c|c|c|c|c|c|}
\hline \multirow{2}{*}{ Factor } & & \multicolumn{3}{|c|}{ New Patients $(n=926)$} & \multicolumn{3}{|c|}{ Re-treatment Patients $(n=236)$} \\
\hline & & $\begin{array}{l}\text { Cases } \\
(n=160)\end{array}$ & $\begin{array}{l}\text { Controls } \\
(\mathrm{n}=766)\end{array}$ & $\begin{array}{l}\text { Stratum specific OR } \\
(95 \% \mathrm{Cl})\end{array}$ & $\begin{array}{l}\text { Cases } \\
(\mathrm{n}=71)\end{array}$ & $\begin{array}{l}\text { Controls } \\
(n=165)\end{array}$ & $\begin{array}{l}\text { Stratum specific OR } \\
95 \% \mathrm{Cl}\end{array}$ \\
\hline \multirow[t]{2}{*}{ Sex } & Male & $100 / 160$ & $398 / 766$ & $1.5(1.1-2.2)$ & $54 / 71$ & $118 / 165$ & $1.3(0.6-2.3)$ \\
\hline & Female & $60 / 160$ & $368 / 766$ & 1.0 & $17 / 71$ & $47 / 165$ & 1.0 \\
\hline \multicolumn{2}{|c|}{ Median age } & 30 & 34 & $p=0.001$ & 33 & 39 & $p=0.003$ \\
\hline
\end{tabular}

The patient category was not documented for one case patient

*One person's age was unknown

some patients did not want other co-workers to know they had TB.

Among new TB patients, cases were more likely than controls to be male, to lack formal education, to have changed residence during TB treatment, to be a laborer, and to have missed treatment due to employment. Among re-treatment TB patients, cases were more likely than controls to have changed residence during TB treatment and to have missed treatment due to employment. New and re-treatment cases and controls were similar in terms of feeling ashamed, seeing a traditional healer, alcohol use, and experiencing side effects (Table 3).

\section{Patient-related factors}

Most patients reported having told someone about their TB diagnosis, and these findings were similar between cases and controls. However, among both new and retreatment groups, cases were more likely than controls to report feeling shame or embarrassment about having TB (Table 3). Defaulters were also more likely than controls to report that they did not feel better with treatment. Among new TB patients, cases were less likely than controls to have felt supported by their family during TB treatment. Reported causes of TB were similar between groups; although, some incorrect causes were commonly cited by both cases and controls including "witchcraft" and "taboo". The majority of cases and controls reported that they originally thought they would have finished taking treatment, felt that TB was curable, and that they could die from TB if not treated.

\section{Therapy and condition-related factors}

New and re-treatment cases and controls were similar in terms of their type of TB (pulmonary vs. extrapulmonary), hospitalization for TB treatment, history of previous treatment failure and having treatment side effects. Among new TB patients, cases were more likely than controls to have taken TB treatment on an empty stomach, to have seen a traditional healer while on treatment for $\mathrm{TB}$ and to use alcohol during treatment. Among re-treatment patients, cases were more likely than controls to have a history of previous default.
Among both groups, cases were more likely than controls to report that treatment did not make them feel better.

\section{Health care team and system-related factors}

Cases and controls were similar in terms of type and location of facility they attended for treatment, their reported access to the clinic, the number of clinics attended, and whether they took their treatment under direct observation. Half of cases and controls reported taking treatment without direct supervision despite the fact that they were all enrolled in DOTS. However, the two groups reported marked differences in opinion about experiences with health services and the health staff during their TB treatment. Cases were more likely than controls to report that clinic hours were inconvenient, that the health care worker $(\mathrm{HCW})$ had a negative attitude towards them, did not treat them with respect, that they did not trust the HCW and that they missed treatment because of poor HCW attitude (Table 4). Cases were also more likely than controls to report that they had not received enough education about $\mathrm{TB}$ at the beginning of treatment, that they were not told why treatment would take 6 or more months and lacked counsel and information about TB treatment in general. Among retreatment patients, cases were more likely than controls to report the food from the clinic would help them finish taking their TB treatment.

Two multivariable models were created, one for new patients and one for re-treatment patients (Table 5). Two risk factors were independently associated with default among both new and re-treatment patients: perception of poor $\mathrm{HCW}$ attitude and changing residence during $\mathrm{TB}$ treatment. Among new patients, additional risk factors associated with default were: not receiving adequate counseling or information during $\mathrm{TB}$ treatment (Adjusted OR 1.9, 95\% CI 1.2-2.8), having no formal education (AOR 2.3, 95\% CI 1.2-4.2), drinking any alcohol during TB treatment (AOR 1.9, 95\% CI 1.2-3.0), feeling ashamed to have TB (AOR 2.0, 95\% CI 1.3-3.0), and seeing a traditional healer during TB treatment (AOR 1.9, 95\% CI 1.1-3.4). Among re-treatment patients risk factors associated with default included: a history of previous TB treatment default (AOR 6.4, 95\% CI 2.9-14.0), not feeling 
Table 2 Social and economic characteristics of cases and controls with TB, stratified by patient category, South Africa, 2002

\begin{tabular}{|c|c|c|c|c|c|c|c|c|c|c|c|c|c|}
\hline \multirow{3}{*}{ Characteristic } & & \multicolumn{5}{|c|}{ New TB Patient $(\mathrm{N}=926)$} & \multicolumn{5}{|c|}{ Re-treatment TB Patient $(\mathrm{N}=236)$} & \multicolumn{2}{|l|}{ Combined } \\
\hline & & \multicolumn{2}{|c|}{ Cases (160) } & \multicolumn{2}{|l|}{$\begin{array}{l}\text { Controls } \\
\text { (766) }\end{array}$} & \multirow[t]{2}{*}{ OR $(95 \% \mathrm{Cl})$} & \multicolumn{2}{|c|}{ Cases (71) } & \multicolumn{2}{|l|}{$\begin{array}{l}\text { Controls } \\
(165)\end{array}$} & \multirow[t]{2}{*}{$\begin{array}{l}\text { OR }(95 \% \\
\text { Cl) }\end{array}$} & \multirow[t]{2}{*}{$\begin{array}{l}\mathrm{OR}^{*}(95 \% \\
\mathrm{Cl})\end{array}$} & \multirow[t]{2}{*}{$\begin{array}{l}\text { AOR† } \\
(95 \% \mathrm{Cl})\end{array}$} \\
\hline & & $\mathrm{n} / \mathrm{N}$ & $\%$ & $\mathrm{n} / \mathrm{N}$ & $\%$ & & $\mathrm{n} / \mathrm{N}$ & $\%$ & $\mathrm{n} / \mathrm{N}$ & $\%$ & & & \\
\hline \multirow[t]{2}{*}{ Country of birth } & RSA & $159 / 160$ & 99 & $745 / 766$ & 97 & $4.5(0.7-186)$ & $71 / 71$ & 10 & $163 / 164$ & 99 & $\mathrm{n} / \mathrm{a}$ & $5.6(0.9-231)$ & $4.9(0.7-36)$ \\
\hline & Outside RSA & $1 / 160$ & 1 & $21 / 766$ & 3 & & $0 / 71$ & 0 & $1 / 164$ & 1 & & & \\
\hline \multirow{2}{*}{$\begin{array}{l}\text { Practices } \\
\text { religion }\end{array}$} & Yes & $148 / 160$ & 93 & $687 / 758$ & 91 & $1.3(0.7-9)$ & $63 / 71$ & 89 & $151 / 162$ & 93 & $0.6(0.2-1.7)$ & $1.0(0.6-1.8)$ & $1.0(0.6-1.7)$ \\
\hline & No & $12 / 160$ & 7 & $71 / 758$ & 8 & & $8 / 71$ & 11 & $11 / 162$ & 7 & & & \\
\hline \multirow[t]{2}{*}{ Race } & Black & $129 / 159$ & 81 & $661 / 762$ & 86 & $0.7(0.4-1.1)$ & $57 / 71$ & 80 & $129 / 164$ & 79 & $1.1(0.5-2.4)$ & $0.7(0.5-1.1)$ & $0.8(0.5-1.1)$ \\
\hline & Other & $30 / 159$ & 19 & $101 / 762$ & 14 & & $14 / 71$ & 20 & $35 / 164$ & 21 & & & \\
\hline \multirow[t]{2}{*}{ Marital status } & $\begin{array}{l}\text { Married or } \\
\text { permanent } \\
\text { relationship }\end{array}$ & $50 / 158$ & 32 & $266 / 763$ & 35 & $0.9(0.6-1.3)$ & $23 / 71$ & 32 & $61 / 164$ & 37 & $0.8(0.4-1.5)$ & $0.9(0.6-1.2)$ & $0.8(0.6-1.2)$ \\
\hline & Single & $108 / 158$ & 68 & $497 / 763$ & 65 & & $48 / 71$ & 68 & $103 / 164$ & 63 & & & \\
\hline \multirow{2}{*}{$\begin{array}{l}\text { Biological } \\
\text { children }\end{array}$} & No children & $31 / 157$ & 20 & $147 / 761$ & 19 & $1.0(0.6-1.6)$ & $21 / 70$ & 30 & $31 / 164$ & 19 & $1.8(0.9-3.7)$ & $1.2(0.9-1.8)$ & $1.2(0.9-1.7)$ \\
\hline & $\geq 1$ child & $126 / 157$ & 80 & $614 / 761$ & 81 & & $49 / 70$ & 70 & $133 / 164$ & 81 & & & \\
\hline \multirow{2}{*}{$\begin{array}{l}\text { Formal } \\
\text { Education }\end{array}$} & None & $22 / 160$ & 14 & $61 / 762$ & 8 & $1.8(1.0-3.1)$ & $5 / 70$ & 7 & $17 / 164$ & 10 & $0.7(0.2-2.0)$ & $1.4(0.9-2.3)$ & $1.4(0.9-2.3)$ \\
\hline & Some & $138 / 160$ & 86 & $701 / 762$ & 92 & & $65 / 70$ & 93 & $147 / 164$ & 90 & & & \\
\hline \multirow[t]{2}{*}{ Owns a house } & Yes & $81 / 157$ & 52 & $386 / 759$ & 51 & $1.0(0.7-1.5)$ & $37 / 71$ & 52 & $89 / 164$ & 54 & $0.9(0.5-1.6)$ & $1.0(0.7-1.5)$ & $1.0(0.7-1.3)$ \\
\hline & No & $76 / 157$ & 48 & $373 / 759$ & 49 & & $34 / 71$ & 48 & $75 / 164$ & 46 & & & \\
\hline \multirow[t]{2}{*}{ Owns cattle } & Yes & $10 / 157$ & 6 & $31 / 755$ & 4 & $1.6(0.7-3.4)$ & $2 / 71$ & 3 & $9 / 164$ & 3 & $\begin{array}{l}0.5(0.05- \\
2.5)\end{array}$ & $1.2(0.6-2.4)$ & $1.2(0.6-2.3)$ \\
\hline & No & $147 / 157$ & 94 & $724 / 755$ & 96 & & $9 / 71$ & 97 & $155 / 164$ & 97 & & & \\
\hline \multirow[t]{2}{*}{ Owns radio } & Yes & $111 / 158$ & 70 & $500 / 759$ & 66 & $1.2(0.8-1.8)$ & $45 / 71$ & 63 & $115 / 164$ & 70 & $0.7(0.4-1.4)$ & $1.1(0.8-1.5)$ & $1.1(0.7-1.5)$ \\
\hline & $\mathrm{No}$ & $47 / 158$ & 30 & $259 / 759$ & 34 & & $26 / 71$ & 27 & $49 / 164$ & 30 & & & \\
\hline \multirow{2}{*}{$\begin{array}{l}\text { Changed } \\
\text { residence } \\
\text { during TB } \\
\text { treatment }\end{array}$} & Yes & 22/158 & 14 & $49 / 756$ & 6 & $2.3(1.3-4.1)$ & 16/71 & 23 & $12 / 165$ & 7 & $3.7(1.5-9.1)$ & $2.8(1.8-4.2)$ & $2.7(1.7-4.2)$ \\
\hline & $\overline{\mathrm{No}}$ & $136 / 158$ & 86 & $707 / 756$ & 94 & & $55 / 71$ & 77 & $153 / 165$ & 93 & & & \\
\hline \multirow[t]{2}{*}{ Employed } & Yes & $81 / 157$ & 52 & $386 / 759$ & 51 & $1.0(0.7-1.5)$ & $37 / 71$ & 52 & $89 / 164$ & 54 & $0.9(0.5-1.6)$ & $1.0(0.7-1.4)$ & $1.0(0.7-1.3)$ \\
\hline & $\overline{\mathrm{No}}$ & $76 / 157$ & 48 & $373 / 759$ & 49 & & $34 / 71$ & 48 & $75 / 164$ & 46 & & & \\
\hline \multirow{2}{*}{$\begin{array}{l}\text { Type of } \\
\text { employment }\end{array}$} & Laborer & $29 / 83$ & 35 & $88 / 370$ & 24 & $1.7(1.0-2.9)$ & $13 / 38$ & 34 & $19 / 81$ & 23 & $1.7(0.7-1.3)$ & $1.7(0.6-4.3)$ & $1.7(1.1-2.6)$ \\
\hline & Other & $54 / 83$ & 65 & $282 / 370$ & 76 & & $25 / 38$ & 66 & $62 / 81$ & 77 & & & \\
\hline \multirow{2}{*}{$\begin{array}{l}\text { Missed } \\
\text { treatment due } \\
\text { to employment }\end{array}$} & Yes & $39 / 85$ & 46 & $16 / 381$ & 4 & 19 (9.6-40) & $14 / 40$ & 35 & $4 / 80$ & 5 & $10(2.8-45)$ & $16(9-31)$ & $16(9-29)$ \\
\hline & $\overline{\mathrm{No}}$ & $46 / 85$ & 54 & $365 / 381$ & 96 & & $26 / 40$ & 65 & $76 / 80$ & 95 & & & \\
\hline \multirow{2}{*}{$\begin{array}{l}\text { In prison during } \\
\text { treatment }\end{array}$} & Yes & $7 / 156$ & 5 & $25 / 748$ & 3 & $1.4(0.5-3.3)$ & $6 / 71$ & 8 & $11 / 165$ & 7 & $1.3(0.4-4.0)$ & $1.5(0.7-2.9)$ & $1.3(0.7-2.6)$ \\
\hline & $\overline{\mathrm{No}}$ & $149 / 156$ & 95 & $723 / 748$ & 97 & & $65 / 71$ & 92 & $154 / 165$ & 93 & & & \\
\hline
\end{tabular}

*Combined bivariate odds ratio

+ Odds ratio adjusted for patient category

better with TB treatment (AOR 21, 95\% CI 5.2-84), and agreement with the statement "food given to me by the nurses at the clinic would better help me finish my TB treatment" (AOR 5.0, 95\% CI 1.3-19).

\section{Discussion}

TB treatment interruption often leads to poor final treatment outcomes and drug-resistance, which has been a challenge for the Department of Health in South 
Table 3 Patient, condition and therapy-related factors of cases and controls with TB, stratified by patient category, South Africa, 2002

\begin{tabular}{|c|c|c|c|c|c|c|c|c|c|c|c|c|c|}
\hline \multirow[b]{3}{*}{ Characteristic } & & \multicolumn{5}{|c|}{ New TB Patient $(\mathrm{N}=926)$} & \multicolumn{5}{|c|}{ Re-treatment TB Patient $(\mathrm{N}=236)$} & \multicolumn{2}{|l|}{ Combined } \\
\hline & & \multicolumn{2}{|c|}{ Cases (160) } & \multicolumn{2}{|l|}{$\begin{array}{l}\text { Controls } \\
\text { (766) }\end{array}$} & \multirow[t]{2}{*}{$\begin{array}{l}\text { OR } \\
(95 \% \mathrm{Cl})\end{array}$} & \multicolumn{2}{|c|}{ Cases $(71)$} & \multicolumn{2}{|l|}{$\begin{array}{l}\text { Controls } \\
\text { (165) }\end{array}$} & \multirow[t]{2}{*}{$\begin{array}{l}\text { OR }(95 \% \\
\text { Cl) }\end{array}$} & \multirow[t]{2}{*}{$\begin{array}{l}\mathrm{OR}^{*}(95 \% \\
\mathrm{Cl})\end{array}$} & \multirow[t]{2}{*}{$\begin{array}{l}\text { AOR† } \\
(95 \% \mathrm{Cl})\end{array}$} \\
\hline & & $\mathrm{n} / \mathrm{N}$ & $\%$ & $\mathrm{n} / \mathrm{N}$ & $\%$ & & $n / N$ & $\%$ & $\mathrm{n} / \mathrm{N}$ & $\%$ & & & \\
\hline \multirow[t]{2}{*}{ Felt ashamed about TB } & Yes & $67 / 155$ & 43 & 181/753 & 24 & $2.4(1.6-3.5)$ & $31 / 71$ & 44 & $48 / 164$ & 30 & $1.9(1.0-3.5)$ & $2.3(1.7-3.1)$ & $2.2(1.6-3.0)$ \\
\hline & No & $88 / 155$ & 67 & $572 / 753$ & 76 & & $40 / 71$ & 56 & $116 / 164$ & 70 & & & \\
\hline \multirow{2}{*}{$\begin{array}{l}\text { Felt supported by } \\
\text { family }\end{array}$} & Yes & $116 / 153$ & 76 & $651 / 736$ & 88 & $0.4(0.2-0.6)$ & $54 / 67$ & 80 & $140 / 160$ & 88 & $0.6(0.3-1.4)$ & $0.5(0.3-0.7)$ & $0.5(0.3-0.7)$ \\
\hline & No & $37 / 153$ & 24 & $85 / 736$ & 12 & & $13 / 67$ & 20 & $20 / 160$ & 22 & & & \\
\hline \multirow{2}{*}{$\begin{array}{l}\text { Knew treatment } \\
\text { duration was } \geq 6 \\
\text { months }\end{array}$} & Yes & $108 / 149$ & 72 & $623 / 741$ & 84 & $0.9(0.4-2.0)$ & $55 / 69$ & 80 & $133 / 163$ & 82 & $0.5(0.3-0.8)$ & $0.6(0.4-0.8)$ & $0.6(0.4-0.8)$ \\
\hline & No & $41 / 149$ & 28 & $118 / 741$ & 16 & & $14 / 69$ & 20 & $30 / 163$ & 18 & & & \\
\hline \multirow{2}{*}{$\begin{array}{l}\text { Felt they might die } \\
\text { from TB }\end{array}$} & Yes & $96 / 154$ & 62 & $511 / 749$ & 68 & $0.8(0.5-1.1)$ & $41 / 65$ & 63 & $118 / 159$ & 74 & $0.6(0.3-1.2)$ & $0.7(0.5-1.0)$ & $0.7(0.5-1.0)$ \\
\hline & No & $58 / 154$ & 38 & $238 / 749$ & 32 & & $24 / 65$ & 37 & $41 / 159$ & 26 & & & \\
\hline \multirow{2}{*}{$\begin{array}{l}\text { Thought they would } \\
\text { finish taking treatment }\end{array}$} & Yes & $112 / 143$ & 78 & $573 / 734$ & 78 & $1.0(0.6-1.6)$ & $52 / 63$ & 63 & $130 / 152$ & 74 & $0.8(0.3-2.0)$ & $1.0(0.7-1.5)$ & $1.0(0.7-1.4)$ \\
\hline & No & $31 / 143$ & 22 & $161 / 734$ & 22 & & $11 / 63$ & 17 & $22 / 152$ & & & & \\
\hline \multirow{2}{*}{$\begin{array}{l}\text { Felt that it is possible } \\
\text { to cure TB }\end{array}$} & Yes & $119 / 123$ & 97 & 729/738 & 99 & $0.4(0.1-1.7)$ & $58 / 62$ & 94 & $149 / 153$ & 97 & $0.4(0.1-2.2)$ & $0.3(0.1-0.9)$ & $0.4(0.2-0.9)$ \\
\hline & No & $4 / 123$ & 3 & 9/738 & 1 & & $4 / 62$ & 6 & $5 / 153$ & 3 & & & \\
\hline \multirow{2}{*}{$\begin{array}{l}\text { Saw a traditional healer } \\
\text { during TB treatment }\end{array}$} & Yes & $30 / 159$ & 19 & $52 / 757$ & 7 & $3.2(1.8-5.3)$ & $9 / 69$ & 13 & $12 / 165$ & 7 & $1.9(0.7-5.2)$ & $2.8(1.7-4.3)$ & $2.8(1.8-4.3)$ \\
\hline & No & $129 / 159$ & 18 & $705 / 757$ & 93 & & $60 / 69$ & 87 & $153 / 165$ & 93 & & & \\
\hline \multirow[t]{2}{*}{ Type of TB } & Pulmonary & $150 / 157$ & 96 & 732/749 & 98 & $0.9(0.2-1.4)$ & $63 / 69$ & 99 & $157 / 161$ & 98 & $\begin{array}{l}0.3(0.05- \\
1.2)\end{array}$ & $0.4(0.2-0.9)$ & $0.4(0.2-0.8)$ \\
\hline & $\begin{array}{l}\text { Extra } \\
\text { Pulmonary }\end{array}$ & $7 / 157$ & 4 & $17 / 749$ & 2 & & $6 / 69$ & 1 & $5 / 161$ & 2 & & & \\
\hline \multirow[t]{2}{*}{ Alcohol use } & Yes & $51 / 154$ & 33 & $157 / 753$ & 21 & $1.9(1.3-2.8)$ & $27 / 70$ & 39 & $46 / 163$ & 28 & $1.6(0.8-2.3)$ & $1.9(1.3-2.6)$ & $1.8(1.3-2.5)$ \\
\hline & No & $103 / 154$ & 77 & $596 / 753$ & 79 & & $43 / 70$ & 61 & $117 / 163$ & 72 & & & \\
\hline \multirow[t]{2}{*}{ Marijuana/mandrax use } & Yes & $6 / 159$ & 4 & $29 / 756$ & 4 & $1.0(0.3-2.5)$ & $8 / 71$ & 11 & $9 / 165$ & 5 & $2.2(0.7-6.7)$ & $1.5(0.7-2.9)$ & $1.4(0.7-2.6)$ \\
\hline & No & $153 / 159$ & 96 & $727 / 756$ & 96 & & $63 / 71$ & 89 & $156 / 165$ & 95 & & & \\
\hline \multirow[t]{2}{*}{ History of default } & No & $\mathrm{n} / \mathrm{a}$ & $\begin{array}{l}\mathrm{n} / \\
\mathrm{a}\end{array}$ & $\mathrm{n} / \mathrm{a}$ & $\begin{array}{l}\mathrm{n} / \\
\mathrm{a}\end{array}$ & $\mathrm{n} / \mathrm{a}$ & $26 / 165$ & 16 & $38 / 71$ & 53 & $6.2(3.1-12)$ & $\mathrm{n} / \mathrm{a}$ & $\mathrm{n} / \mathrm{a}$ \\
\hline & Yes & $\mathrm{n} / \mathrm{a}$ & $\begin{array}{l}\mathrm{n} / \\
\mathrm{a}\end{array}$ & $\mathrm{n} / \mathrm{a}$ & $\begin{array}{l}\mathrm{n} / \\
\mathrm{a}\end{array}$ & $\mathrm{n} / \mathrm{a}$ & $139 / 165$ & 84 & $33 / 71$ & 47 & & & \\
\hline \multirow{2}{*}{$\begin{array}{l}\text { Hospitalized for TB } \\
\text { treatment }\end{array}$} & Yes & $54 / 155$ & 35 & $287 / 758$ & 38 & $0.9(0.6-1.1)$ & $25 / 70$ & 36 & $66 / 163$ & 40 & $0.8(0.4-1.5)$ & $0.9(0.6-1.2)$ & $0.9(0.6-1.2)$ \\
\hline & No & $101 / 155$ & 65 & $471 / 758$ & 62 & & $45 / 70$ & 64 & $97 / 163$ & 60 & & & \\
\hline \multirow[t]{2}{*}{$\begin{array}{l}\text { Felt better with } \\
\text { treatment }\end{array}$} & No & $32 / 150$ & 21 & $15 / 754$ & 2 & $13.3(7.0-27)$ & $18 / 67$ & 27 & $5 / 162$ & 3 & $\begin{array}{l}11.5(3.9- \\
41)\end{array}$ & $\begin{array}{l}13.3(7.5- \\
24)\end{array}$ & $\begin{array}{l}12.7(7.3- \\
22)\end{array}$ \\
\hline & Yes & $118 / 150$ & 79 & 739/754 & 98 & & $49 / 67$ & 73 & $157 / 162$ & 97 & & & \\
\hline Treatment side effects & Yes & $73 / 138$ & 53 & $297 / 679$ & 44 & $1.4(1.0-2.2)$ & $39 / 60$ & 65 & $79 / 152$ & 52 & $1.7(0.9-7.8)$ & $1.6(1.2-2.2)$ & $1.5(1.1-2.1)$ \\
\hline & No & $65 / 138$ & 47 & $382 / 679$ & 56 & & $21 / 60$ & 35 & $73 / 152$ & 48 & & & \\
\hline$\geq 1 \mathrm{da}$ & Yes & 28/155 & 18 & $98 / 759$ & 13 & $1.5(0.9-2.4)$ & $16 / 70$ & 23 & $30 / 163$ & 18 & $1.3(0.6-2.7)$ & $1.5(1.0-2.2)$ & $1.4(0.9-2.1)$ \\
\hline & $\overline{\mathrm{No}}$ & $127 / 155$ & 82 & $661 / 759$ & 87 & & $54 / 70$ & 77 & $133 / 163$ & 82 & & & \\
\hline Took TB treatment on & Yes & $49 / 142$ & 35 & $183 / 725$ & 25 & $1.6(1.0-2.3)$ & $24 / 62$ & 39 & $43 / 160$ & 27 & $1.7(0.9-3.3)$ & $1.6(1.2-2.3)$ & $1.6(1.2-2.2)$ \\
\hline & $\mathrm{No}$ & $93 / 142$ & 65 & $542 / 729$ & 75 & & $38 / 62$ & 61 & $117 / 160$ & 73 & & & \\
\hline
\end{tabular}

*Combined bivariate odds ratio

† Odds ratio adjusted by patient category 
Table 4 Health care team and system related factors, cases and controls with TB, stratified by patient category, South Africa, 2002

\begin{tabular}{|c|c|c|c|c|c|c|c|c|c|c|c|c|c|}
\hline \multirow[b]{3}{*}{ Factor } & & \multicolumn{5}{|c|}{ New TB Patient $(\mathrm{N}=926)$} & \multicolumn{5}{|c|}{ Re-treatment TB Patient $(\mathrm{N}=236)$} & \multicolumn{2}{|l|}{ Combined } \\
\hline & & \multicolumn{2}{|c|}{ Cases (160) } & \multicolumn{2}{|l|}{$\begin{array}{l}\text { Controls } \\
\text { (766) }\end{array}$} & \multirow[t]{2}{*}{$\begin{array}{l}\text { OR(95\% } \\
\text { Cl) }\end{array}$} & \multicolumn{2}{|c|}{ Cases $(71)$} & \multicolumn{2}{|l|}{$\begin{array}{l}\text { Controls } \\
(165)\end{array}$} & \multirow[t]{2}{*}{$\begin{array}{l}\text { OR(95\% } \\
\text { Cl) }\end{array}$} & \multirow[t]{2}{*}{$\begin{array}{l}\mathrm{OR}^{*}(95 \% \\
\mathrm{Cl})\end{array}$} & \multirow[t]{2}{*}{$\begin{array}{l}\text { AOR† } \\
(95 \% \mathrm{Cl})\end{array}$} \\
\hline & & $\mathrm{n} / \mathrm{N}$ & $\%$ & $n / N$ & $\%$ & & $\mathrm{n} / \mathrm{N}$ & $\%$ & $\mathrm{n} / \mathrm{N}$ & $\%$ & & & \\
\hline \multirow[t]{2}{*}{ Clinic hours convenient } & No & $32 / 147$ & 22 & $60 / 747$ & 8 & $3.2(1.9-5.2)$ & $12 / 64$ & 19 & $8 / 160$ & 5 & $4.4(1.5-13)$ & $3.2(2.1-5.0)$ & $3.4(2.2-5.2)$ \\
\hline & Yes & $115 / 147$ & 78 & $687 / 747$ & 92 & & $52 / 64$ & 81 & $152 / 160$ & 95 & & & \\
\hline \multirow[t]{2}{*}{ Time spent waiting at clinic } & $\begin{array}{l}\leq 1 \\
\text { hour }\end{array}$ & $15 / 146$ & 10 & $74 / 709$ & 10 & $1.0(0.5-1.8)$ & $9 / 66$ & 14 & $13 / 154$ & 8 & $1.7(0.6-4.6)$ & $1.1(0.7-1.9)$ & $1.1(0.7-1.9)$ \\
\hline & $\begin{array}{l}>1 \\
\text { hour }\end{array}$ & $131 / 146$ & 90 & $635 / 709$ & 90 & & $57 / 66$ & 86 & $141 / 154$ & 92 & & & \\
\hline \multirow{2}{*}{$\begin{array}{l}\text { Did HCW treat you with } \\
\text { respect? }\end{array}$} & No & $11 / 158$ & 7 & $19 / 761$ & 3 & $2.9(1.2-6.6)$ & $10 / 71$ & 14 & $3 / 165$ & 2 & $8.9(2.1-51)$ & $4.1(2.1-8.0)$ & $4.1(2.2-7.8)$ \\
\hline & Yes & $147 / 158$ & 93 & $742 / 761$ & 97 & & $61 / 71$ & 86 & $162 / 165$ & 98 & & & \\
\hline \multirow[t]{2}{*}{ HCW attitude } & Poor & $17 / 160$ & 11 & $30 / 763$ & 4 & $2.9(1.5-5.6)$ & $12 / 71$ & 17 & $5 / 164$ & 3 & $6.5(2.0-24)$ & $3.6(2.1-6.3)$ & $3.7(2.2-6.2)$ \\
\hline & Good & $143 / 160$ & 89 & 733/763 & 96 & & $59 / 71$ & 83 & $159 / 164$ & 97 & & & \\
\hline \multirow[t]{2}{*}{$\begin{array}{l}\text { Missed treatment due to HCW } \\
\text { attitude }\end{array}$} & Yes & $15 / 155$ & 10 & $19 / 763$ & 25 & $4.2(1.9-8.9)$ & $9 / 69$ & 13 & $1 / 162$ & 1 & $\begin{array}{l}24.2(3.1- \\
1064)\end{array}$ & $5.4(2.8-11)$ & $5.8(3.0-11)$ \\
\hline & No & $140 / 155$ & 90 & $744 / 763$ & 75 & & $60 / 69$ & 87 & $161 / 162$ & 99 & & & \\
\hline \multirow[t]{2}{*}{ Trusted HCW } & No & $9 / 156$ & 6 & $14 / 749$ & 2 & $3.2(1.2-8.1)$ & $5 / 66$ & 8 & $4 / 163$ & 25 & $3.3(0.7-17)$ & $3.3(1.5-7.2)$ & $3.2(1.6-6.7)$ \\
\hline & Yes & $147 / 156$ & 94 & 735/749 & 98 & & $61 / 66$ & 92 & 159/163 & 75 & & & \\
\hline \multirow{2}{*}{$\begin{array}{l}\text { Told why TB treatment must } \\
\text { be taken } \geq 6 \text { months }\end{array}$} & No & $73 / 152$ & 48 & $225 / 746$ & 30 & $2.1(1.5-3.1)$ & $31 / 70$ & 44 & $47 / 162$ & 29 & $1.9(1.0-3.6)$ & $2.0(1.5-2.8)$ & $2.1(1.5-3.1)$ \\
\hline & Yes & $79 / 152$ & 52 & $521 / 746$ & 70 & & $39 / 70$ & 56 & $115 / 162$ & 71 & & & \\
\hline \multirow{2}{*}{$\begin{array}{l}\text { Given counsel or information } \\
\text { on TB treatment }\end{array}$} & No & $53 / 155$ & 34 & $163 / 756$ & 22 & $1.9(1.3-2.8)$ & $27 / 69$ & 39 & $40 / 164$ & 24 & $2.0(1.0-3.8)$ & $1.9(1.4-2.7)$ & $1.9(1.4-2.6)$ \\
\hline & Yes & $102 / 155$ & 26 & $593 / 756$ & 78 & & $42 / 69$ & 61 & $124 / 164$ & 76 & & & \\
\hline \multirow{2}{*}{$\begin{array}{l}\text { Received enough education } \\
\text { about TB at beginning of } \\
\text { treatment }\end{array}$} & No & $17 / 98$ & 17 & $44 / 592$ & 7 & $2.6(1.3-4.9)$ & $10 / 39$ & 26 & $3 / 121$ & 2 & $\begin{array}{l}13.6(3.1- \\
80)\end{array}$ & $3.5(2.0-5.9)$ & $3.6(2.1-6.2)$ \\
\hline & Yes & $81 / 98$ & 83 & $548 / 592$ & 93 & & $29 / 39$ & 74 & $118 / 121$ & 88 & & & \\
\hline \multirow{2}{*}{$\begin{array}{l}\text { Took pills without direct } \\
\text { supervision (DOT) }\end{array}$} & Yes & $77 / 148$ & 52 & $400 / 741$ & 54 & $0.9(0.6-1.3)$ & $32 / 70$ & 46 & $55 / 160$ & 34 & $1.6(0.8-3.0)$ & $1.0(0.7-1.3)$ & $1.1(0.8-1.4)$ \\
\hline & No & $71 / 148$ & 48 & $341 / 741$ & 46 & & $38 / 70$ & 54 & $105 / 160$ & 66 & & & \\
\hline \multirow{2}{*}{$\begin{array}{l}\text { Agreed that if food was } \\
\text { provided by clinic, adherence } \\
\text { to treatment would have } \\
\text { been better }\end{array}$} & Yes & $114 / 139$ & 82 & $553 / 688$ & 80 & $1.1(0.6-1.9)$ & $63 / 68$ & 93 & $114 / 151$ & 76 & $4.1(1.4-14)$ & $1.5(1.0-2.4)$ & $1.5(1.0-2.3)$ \\
\hline & $\mathrm{No}$ & $25 / 139$ & 18 & $135 / 688$ & 20 & & $5 / 68$ & 7 & $37 / 151$ & 24 & & & \\
\hline
\end{tabular}

*Combined bivariate odds ratio

† Odds ratio adjusted by patient category

Africa for more than two decades. In our case control study of TB patients treated in 2002, only $26 \%$ of cases and $43 \%$ of controls could be traced and is an important limitation of the study. Nevertheless, we identified several potentially modifiable risk factors associated with treatment default. Notably, we found an association between default and poor perceived relations between the HCW and patient. The importance of this relationship and its influence on adherence to treatment is well established. A few studies have reported specifically on patient's satisfaction with health worker attitude and service provision and risk of default [6,11,14-16]. Vijay et. al found similar poor provider patient relationship factors associated with default among new smear positive TB patients [16]. Some studies have reported communication barriers or poor communication between patients and providers as being linked to poor adherence $[15,17,18]$. Perceived communication quality or lack thereof may reflect the status of the patient provider relationship and influence patient behavior $[18,19]$. Other studies have reported associations between TB treatment default and a poor level of knowledge of TB 
Table 5 Multivariable logistic regression analysis for TB treatment default, stratified by patient category (new, retreatment), South Africa, 2002*

\begin{tabular}{|c|c|c|c|c|}
\hline \multirow[b]{2}{*}{ Risk Factor } & \multicolumn{2}{|c|}{ New Patients (848) } & \multicolumn{2}{|c|}{ Re-treatment Patients (211) } \\
\hline & Adjusted OR & $95 \% \mathrm{Cl}$ & Adjusted OR & $95 \% \mathrm{Cl}$ \\
\hline Poor HCW attitude & 2.1 & $1.1-4.4$ & 12 & $2.2-66$ \\
\hline Changing residence during TB treatment & 2.0 & $1.1-3.7$ & 3.4 & $1.1-9.9$ \\
\hline Did not receive adequate counseling or information & 1.9 & $1.2-2.8$ & & \\
\hline No formal education & 2.3 & $1.2-4.2$ & & \\
\hline Drank any alcohol during treatment & 1.9 & $1.2-3.0$ & & \\
\hline Felt ashamed to have TB & 2.0 & 1.3-3.0 & & \\
\hline Saw a traditional healer for TB & 1.9 & $1.1-3.4$ & & \\
\hline History of previous TB default & & & 6.4 & $2.9-14$ \\
\hline Felt better with treatment & & & 21 & $5.2-84$ \\
\hline Agreed that if food was provided by clinic, adherence to treatment would have been better & & & 5.0 & $1.3-19$ \\
\hline
\end{tabular}

*Models adjusted for age and sex; other variables considered but not included in the model: race, marital status, employment status, rural or urban setting

or low patient satisfaction regarding information provided concerning their illness [17-20]. Patient education materials should be appropriately tailored to educationlevel and a non-literate audience as needed.

Ensuring continuity of TB services among patients who are mobile is challenging. Our findings that patient mobility (changing residence) was associated with default underscores the need for improved communication and coordination between the patient and health services. Holtz, et. al., also found that changing residence was independently associated with MDR TB treatment default among MDR TB patients in South Africa [6]. The association between migration and default has been cited in other settings $[9,20]$. Patients require education about the ability to transfer care and TB programs should to be designed to closely follow-up and effectively refer patients to alternate DOTS treatment sites in a timely manner when required.

Among new TB patients, feeling shame was associated with default. Qualitative and quantitative studies among patients and providers have identified TB stigma as a likely barrier to adherence [17,21-23]. The growth of the HIV and TB co-epidemic and the well known relationship between the two diseases may have intensified the problem of TB stigma in South Africa [24]. Successful interventions such as community education and integrating community involvement in TB control may help to reduce social barriers to treatment and stigma $[25,26]$.

Alcohol use was also associated with default among new TB patients. Alcohol use or alcohol abuse has been frequently reported as a risk factor for default $[8,9,16,27-30]$. While it can be difficult for patients to change this behavior, effective primary care behavior interventions to reduce patient alcohol use have been demonstrated [31,32].
The use of traditional healers is common in South Africa. Among new patients, use of traditional healers during TB treatment was associated with default. Several reports have described how seeking care from traditional healers can delay prompt diagnosis and treatment for $\mathrm{TB}$ and can negatively influence morbidity and mortality from TB [33-35]. Some TB case finding and treatment models have successfully partnered with traditional healers to implement community-based DOTS with good outcomes [36,37]. Every effort should be made to expand these successful projects, educate and collaborate with traditional healers and involve them in $\mathrm{TB}$ control.

An association between a previous history of default and subsequent default was found in our study and has been reported in several other settings [38-41]. Efforts to prevent first-time default among new patients, and methods to rapidly identify and intervene with previous defaulters who are at high risk are needed. As "felt better with treatment" was also associated with default among re-treatment patients, every effort should be made to monitor response to therapy and manage potential concurrent illnesses in an integrated and timely fashion.

It is concerning that half of all patients reported that they were not supervised when they took their TB treatment. Implementation of DOT services has been a challenge in South Africa [42]. Ntshanga, et. al., conducted an evaluation of the DOTS program in crisis districts in KwaZulu-Natal and showed poor implementation of DOT where low coverage, low quality and high caseloads were associated with poorer outcomes [43].

The general health care crisis, shortage of health workers and increased health care burden (largely from the HIV epidemic) in South Africa has led to compromises in quality of care, inadequate service delivery and 
is reflected in our study findings. The variety of factors associated with TB treatment default identified, touching upon each of the classic adherence domains, suggests a multi-pronged approach is needed.

Successful small scale comprehensive treatment models to improve TB treatment adherence have been evaluated and documented [43-47]. Key elements of these models include: (1) enhanced training for HCWs in communication and counseling skills, emphasizing patient-centered approaches; (2) development and use of effective and appropriate patient education materials, with an emphasis on patient adherence and self-monitoring tools; (3) close clinical follow-up; (4) effective incentives; (5) social assistance programs; (6) improved community participation, providing flexibility in DOT provider and decentralizing treatment through community based DOTS; (7) integrated TB and HIV care; (8) enhanced program supervision and management; and (9) adequate financial and human resources.

There were several limitations of this study. The low response rate limits power to draw generalized conclusions about South Africa. Other retrospective case control studies have reported similar difficulties in locating patients after they have left care at the TB program, especially defaulters $[6,15-17,48-50]$. The retrospective nature of the study and self-reported data collected from patients is subject to recall bias which may reduce the risk estimate. Cases interviewed were more likely to default later in the treatment course than those not interviewed. Because there is an unknown temporal association between the risk factors identified and treatment default, this limits our ability to potentially identify time-points in case management at which different risk factors for non-adherence are more important and where specific types of adherence strategies may have an increased impact.

Lastly, there are inherent difficulties in collecting subjective data between such disparate subjects as social factors, economic factors, health care team characteristics, and patient-level factors all reported through one mechanism, the patient self-report. The self-report refers to experience, rather than to reality. It is the experience (of reality and not reality itself) that informs future decisions by people about adherence and retention. Hence if we are interested in understanding the relationship between satisfaction and adherence, we need to know patient subjective experiences. The same applies to experiences that are quantifiable and can actually be verified from institutional data, such as the number of doctors seen, waiting times, etc. Also here the subjective experience is the overarching factor when it comes to making decisions by patients. Some of these factors we measured have direct impact on health care services, and others have indirect but equally large impacts on services. Unfortunately it was also not possible to include the provider perspective (health care workers and treatment supporters) on default and non-adherence in this study.

\section{Conclusion}

Standard 9 of the International Standards of Tuberculosis Care includes creating a treatment environment to promote adherence and monitoring results using a patient centered approach and fostering respect between the patient and provider [51]. There are now several small scale models incorporating patient-centered approaches showing success at achieving good TB treatment outcomes in local settings in South Africa and other countries. Adequate resources, good program management, supervision and ongoing evaluation are urgently needed to ensure successful scale up and sustainability of these programs. While South Africa faces challenges of an overburdened health system amidst the escalating TB/HIV co-epidemic, the country has made policy changes and begun implementing innovative ways to reduce staff shortages, improve staff motivation, develop new approaches to clinical practice and education [52]. These promising changes provide a fertile ground on which to institute interventions to reduce default from $\mathrm{TB}$ treatment.

\section{Competing interest statement}

The authors declare that they have no competing interests.

\section{Abbreviations}

HIV: Human immunodeficiency virus; HCW: Health care worker; TB: tuberculosis.

\section{Acknowledgements}

We would like to thank the eight provincial Departments of Health for their approval and assistance in conducting this study. We also thank Charles D. Wells, Kayla F. Laserson, Malebo Pooe, Peta Davis, Joanne Kirsten, William Coggin all of whom played important roles in facilitating and guiding the planning and implementation of this study. We thank all of our study interviewers who worked so hard to trace patients and conduct interviews. The study was entirely financed by the United States Agency of International Development (USAID), a U.S. government agency providing economic and humanitarian assistance. The funders had no role in study design, data collection and analysis, decision to publish, or preparation of the manuscript.

\section{Author details}

'Division of Tuberculosis Elimination, Centers for Disease Control and Prevention, 1600 Clifton Road NE, Atlanta, GA 30333, USA. ²Tuberculosis Epidemiology and Intervention Research Unit, Medical Research Council, 1 Soutpansberg Road, Pretoria 0001, South Africa. ${ }^{3}$ Stop TB Department, World Health Organization, 20 Avenue Appia, CH-1211 Geneva 27, Switzerland.

\section{Authors' contributions}

$\mathrm{TH}, \mathrm{KW}$, and AM contributed to the conception and design of the study. AF, $J$, and $A M$ lead the acquisition of data. AF, JL, TH, KW, and MvdW actively participated in the analysis and interpretation of data. AF, JL, and $T H$ wrote the first draft of the manuscript, and KW, AM, and MvdW provided critical revision of manuscript. All authors read and approved the final manuscript. 
Received: 22 August 2011 Accepted: 20 January 2012

Published: 20 January 2012

\section{References}

1. WHO: Global Tuberculosis Control: Surveillance, Planning, Financing. WHO Report 2004 Geneva, Switzerland; 2004, ISBN 9241562641.

2. Statistics South Africa: Mortality and causes of death in South Africa, 1997-2003; Findings from death notification (Statistical Release P0309.3). 2005 [http://www.statssa.gov.za], Accessed on 15 May 2011.

3. Pablos-Mendez A, Knirsch CA, Barr RG, Lerner BH, Frieden TR: Nonadherence in tuberculosis treatment: predictors and consequences in New York City. Am J Med 1997, 102(2):164-170.

4. Connolly C, Davies GR, Wilkinson D: Who fails to complete tuberculosis treatment? Temporal trends and risk factors for treatment interruption in a community-based directly observed therapy programme in a rural district of South Africa. Int J Tuberc Lung Dis 1999, 3(12):1081-1087.

5. Kharsany AB, Connolly C, Olowolagba A, Abdool Karim SS, Abdool Karim Q: TB treatment outcomes following directly-observed treatment at an urban outpatient specialist TB facility in South Africa. Trop Doct 2006, 36(1):23-25.

6. Holtz TH, Lancaster J, Laserson KF, Wells CD, Thorpe L, Weyer K: Risk factors associated with default from multidrug-resistant tuberculosis treatment, South Africa, 1999-2001. Int J Tuberc Lung Dis 2006, 10(6):649-655.

7. Castelnuovo B: A review of compliance to anti tuberculosis treatment and risk factors for defaulting treatment in Sub Saharan Africa. Afr Health Sci 2010, 10(4):320-324

8. Santha T, Garg R, Frieden TR, Chandrasekaran V, Subramani R, Gopi PG, Selvakumar N, Ganapathy S, Charles N, Rajamma J, et al: Risk factors associated with default, failure and death among tuberculosis patients treated in a DOTS programme in Tiruvallur District, South India, 2000. Int J Tuberc Lung Dis 2002, 6(9):780-788.

9. Jaggarajamma K, Sudha G, Chandrasekaran V, Nirupa C, Thomas A, Santha T, Muniyandi M, Narayanan PR: Reasons for non-compliance among patients treated under Revised National Tuberculosis Control Programme (RNTCP), Tiruvallur district, south India. Indian J Tuberc 2007, 54(3):130-135.

10. Chandrasekaran V, Gopi PG, Santha T, Subramani R, Narayanan PR: Status of re-registered patients for tuberculosis treatment under DOTS programme. Indian J Tuberc 2007, 54(1):12-16.

11. WHO: Adherence to long-term therapies: evidence for action Geneva: World Health Organization; 2003.

12. Lauristen JM, Bruus M: A comprehensive tool for validated entry and documentation of data. EpiData Entry. v.3.1 edition. Odense, Denmark: The EpiData Association; 2006.

13. StataCorp: Statistical Softward: Release 9.0. College Station, TX: Stata Corporation; 2008

14. Hane F, Thiam S, Fall AS, Vidal L, Diop AH, Ndir M, Lienhardt C: Identifying barriers to effective tuberculosis control in Senegal: an anthropological approach. Int J Tuberc Lung Dis 2007, 11(5):539-543.

15. Mishra P, Hansen EH, Sabroe S, Kafle KK: Adherence is associated with the quality of professional-patient interaction in Directly Observed Treatment Short-course, DOTS. Patient Educ Couns 2006, 63(1-2):29-37.

16. Vijay S, Kumar P, Chauhan LS, Vollepore BH, Kizhakkethil UP, Rao SG: Risk factors associated with default among new smear positive TB patients treated under DOTS in India. PLOS ONE 2010, 5(4):e10043.

17. Comolet TM, Rakotomalala R, Rajaonarioa H: Factors determining compliance with tuberculosis treatment in an urban environment, Tamatave, Madagascar. Int J Tuberc Lung Dis 1998, 2(11):891-897.

18. Munro S, Lewin S, Swart T, Volmink J: A review of health behaviour theories: how useful are these for developing interventions to promote long-term medication adherence for TB and HIV/AIDS? BMC Public Health 2007, 7:104.

19. Driver CR, Matus SP, Bayuga S, Winters Al, Munsiff SS: Factors associated with tuberculosis treatment interruption in New York City. J Public Health Manag Pract 2005, 11(4):361-368.

20. Kapella BK, Anuwatnonthakate A, Komsakorn S, Moolphate S, Charusuntonsri P, Limsomboon P, Wattanaamornkiat W, Nateniyom S, Varma JK: Directly observed treatment is associated with reduced default among foreign tuberculosis patients in Thailand. Int J Tuberc Lung Dis 2009, 13(2):232-237.
21. Naidoo P, Dick J, Cooper D: Exploring tuberculosis patients' adherence to treatment regimens and prevention programs at a public health site. Qual Health Res 2009, 19(1):55-70.

22. Suri A, Gan K, Carpenter S: Voices from the field: perspectives from community health workers on health care delivery in rural KwaZuluNatal, South Africa. J Infect Dis 2007, 196(Suppl 3):S505-S511.

23. Edginton ME, Sekatane CS, Goldstein SJ: Patients' beliefs: do they affect tuberculosis control? A study in a rural district of South Africa. Int $J$ Tuberc Lung Dis 2002, 6(12):1075-1082.

24. Rowe KA, Makhubele B, Hargreaves JR, Porter JD, Hausler HP, Pronyk PM Adherence to TB preventive therapy for HIV-positive patients in rural South Africa: implications for antiretroviral delivery in resource-poor settings? Int J Tuberc Lung Dis 2005, 9(3):263-269.

25. Garner $P$, Smith H, Munro S, Volmink J: Promoting adherence to tuberculosis treatment. Bull World Health Organ 2007, 85(5):404-406.

26. Getahun H, Maher D: Contribution of 'TB clubs' to tuberculosis control in a rural district in Ethiopia. Int J Tuberc Lung Dis 2000, 4(2):174-178.

27. Jakubowiak WM, Bogorodskaya EM, Borisov SE, Danilova ID, Kourbatova EV: Risk factors associated with default among new pulmonary TB patients and social support in six Russian regions. Int J Tuberc Lung Dis 2007, 11(1):46-53

28. Hasker E, Khodjikhanov M, Usarova S, Asamidinov U, Yuldashova U, van der Werf MJ, Uzakova G, Veen J: Default from tuberculosis treatment in Tashkent, Uzbekistan; who are these defaulters and why do they default? BMC Infect Dis 2008, 8:97.

29. Gelmanova IY, Keshavjee S, Golubchikova VT, Berezina VI, Strelis AK, Yanova GV, Atwood S, Murray M: Barriers to successful tuberculosis treatment in Tomsk, Russian Federation: non-adherence, default and the acquisition of multidrug resistance. Bull World Health Organ 2007, 85(9):703-711

30. Bhagat VM, Gattani PL: Factors affecting tuberculosis retreatment defaults in Nanded, India. Southeast Asian J Trop Med Public Health 2010, 41(5):1153-1157

31. Kaner EF, Beyer F, Dickinson HO, Pienaar E, Campbell F, Schlesinger C, Heather N, Saunders J, Burnand B: Effectiveness of brief alcohol interventions in primary care populations. Cochrane Database Syst Rev 2007, 2: CD004148

32. Whitlock EP, Polen MR, Green CA, Orleans T, Klein J: Behavioral counseling interventions in primary care to reduce risky/harmful alcohol use by adults: a summary of the evidence for the U.S. Preventive Services Task Force. Ann Intern Med 2004, 140(7):557-568.

33. Banerjee A, Harries AD, Nyirenda T, Salaniponi FM: Local perceptions of tuberculosis in a rural district in Malawi. Int J Tuberc Lung Dis 2000, 4(11):1047-1051.

34. Brouwer JA, Boeree MJ, Kager P, Varkevisser CM, Harries AD: Traditional healers and pulmonary tuberculosis in Malawi. Int J Tuberc Lung Dis 1998, 2(3):231-234

35. Barker RD, Millard FJ, Malatsi J, Mkoana L, Ngoatwana T, Agarawal S, de Valliere S: Traditional healers, treatment delay, performance status and death from TB in rural South Africa. Int J Tuberc Lung Dis 2006, 10(6):670-675

36. Harper ME, Hill PC, Bah AH, Manneh K, McAdam KP, Lienhardt C. Traditional healers participate in tuberculosis control in The Gambia. Int J Tuberc Lung Dis 2004, 8(10):1266-1268.

37. Colvin M, Gumede L, Grimwade K, Maher D, Wilkinson D: Contribution of traditional healers to a rural tuberculosis control programme in Hlabisa, South Africa. Int J Tuberc Lung Dis 2003, 7(9 Suppl 1):S86-S91.

38. Pinidiyapathirage J, Senaratne $W$, Wickremasinghe R: Prevalence and predictors of default with tuberculosis treatment in Sri Lanka. Southeast Asian J Trop Med Public Health 2008, 39(6):1076-1082

39. Chang KC, Leung CC, Tam CM: Risk factors for defaulting from antituberculosis treatment under directly observed treatment in Hong Kong. Int J Tuberc Lung Dis 2004, 8(12):1492-1498.

40. Chan-Yeung M, Noertjojo K, Leung CC, Chan SL, Tam CM: Prevalence and predictors of default from tuberculosis treatment in Hong Kong. Hong Kong Med J 2003, 9(4):263-268.

41. Jha UM, Satyanarayana S, Dewan PK, Chadha S, Wares F, Sahu S, Gupta D, Chauhan LS: Risk factors for treatment default among re-treatment tuberculosis patients in India, 2006. PLOS ONE 2010, 5(1):e8873.

42. Weyer K: Case study: South Africa. Bull World Health Organ 2007, 85(5):391. 
43. Ntshanga SP, Rustomjee R, Mabaso ML: Evaluation of directly observed therapy for tuberculosis in KwaZulu-Natal, South Africa. Trans $R$ Soc Trop Med Hyg 2009, 103(6):571-574.

44. Clarke M, Dick J, Zwarenstein M, Lombard CJ, Diwan VK: Lay health worker intervention with choice of DOT superior to standard TB care for farm dwellers in South Africa: a cluster randomised control trial. Int I Tuberc Lung Dis 2005, 9(6):673-679.

45. Dick J, Lombard C: Shared vision-a health education project designed to enhance adherence to anti-tuberculosis treatment. Int J Tuberc Lung Dis 1997, 1(2):181-186

46. Thiam S, LeFevre AM, Hane F, Ndiaye A, Ba F, Fielding KL, Ndir M, Lienhardt C: Effectiveness of a strategy to improve adherence to tuberculosis treatment in a resource-poor setting: a cluster randomized controlled trial. JAMA 2007, 297(4):380-386.

47. Gandhi NR, Moll AP, Lalloo U, Pawinski R, Zeller K, Moodley P, Meyer E, Friedland G: Successful integration of tuberculosis and HIV treatment in rural South Africa: the Sizonq'oba study. J Acquir Immune Defic Syndr 2009, 50(1):37-43.

48. Vree M, Huong NT, Duong BD, Sy DN, Van le N, Co NV, Cobelens FG, Borgdorff MW: Mortality and failure among tuberculosis patients who did not complete treatment in Vietnam: a cohort study. BMC Public Health 2007, 7:134.

49. Botha E, Den Boon S, Verver S, Dunbar R, Lawrence KA, Bosman M, Enarson DA, Toms I, Beyers N: Initial default from tuberculosis treatment: how often does it happen and what are the reasons? Int I Tuberc Lung Dis 2008, 12(7):820-823.

50. Korenromp EL, Bierrenbach AL, Williams BG, Dye C: The measurement and estimation of tuberculosis mortality. Int I Tuberc Lung Dis 2009, 13(3):283-303.

51. Hopewell PC, Pai M, Maher D, Uplekar M, Raviglione MC: International standards for tuberculosis care. Lancet Infect Dis 2006, 6(11):710-725.

52. Bateman ED, Feldman C, Mash R, Fairall LR, English RG, Jithoo A: Systems for the management of respiratory disease in primary care - an international series: South Africa. Prim Care Respir J 2009, 18(2):69-75.

\section{Pre-publication history}

The pre-publication history for this paper can be accessed here: http://www.biomedcentral.com/1471-2458/12/56/prepub

doi:10.1186/1471-2458-12-56

Cite this article as: Finlay et al: Patient- and provider-level risk factors associated with default from tuberculosis treatment, South Africa, 2002: a case-control study. BMC Public Health 2012 12:56.

\section{Submit your next manuscript to BioMed Central and take full advantage of:}

- Convenient online submission

- Thorough peer review

- No space constraints or color figure charges

- Immediate publication on acceptance

- Inclusion in PubMed, CAS, Scopus and Google Scholar

- Research which is freely available for redistribution 